\title{
Retraction: Crucial role of interleukin-7 in T helper type 17 survival and expansion in autoimmune disease
}

Xuebin Liu, Stewart Leung, Chunxia Wang, Zhu Tan, Ji Wang, Taylor B Guo, Lei Fang, Yonggang Zhao, Bing Wan, Xia Qin, Limin Lu, Runsheng Li, Heng Pan, Mingjuan Song, Ailian Liu, Jian Hong, Hongtao Lu \& Jingwu Z Zhang

Nat. Med. 16, 191-197 (2010); published online 10 January 2010; retracted 5 December 2013

The above manuscript was authored by scientists from the GlaxoSmithKline (GSK) Research and Development Center in Shanghai, China, and a researcher from Baylor Medical College who later became a GSK employee. Following anonymous reports of inaccuracies in this study, GSK conducted an investigation into these allegations.

The investigation established that the data depicted in Figure 6 and in Supplementary Figure 7 were erroneously attributed to experiments at Baylor Medical College with blood cells from patients with multiple sclerosis. In fact, no data from experiments with blood cells from patients with multiple sclerosis and no data from experiments at Baylor Medical College were included in the paper. GSK has therefore concluded that the paper contains erroneous data and requests that it be retracted.

Nature Medicine wishes to acknowledge that the two graphs at the bottom left-hand corner of Figure 2a are identical and that this was an error introduced by the journal during the production of the article. Below are the correct graphs as submitted by the authors (Fig. 1).

All authors agreed to the retraction of the paper with the following exceptions and clarifications. Xuebin Liu and Stewart Leung declined to sign the retraction and stand by the conclusions of the paper. Chunxia Wang, Xia Qin and Limin Lu did not respond to Nature Medicine's requests for comment on the retraction. Ji Wang, Lei Fang, Bing Wan, Jian Hong and Hongtao Lu could not be reached by the journal for comment on the retraction. However, Chunxia Wang, Xia Qin, Limin Lu, Ji Wang, Lei Fang, Bing Wan, Jian Hong and Hongtao Lu signed an initial version of the retraction submitted to the journal by GSK.

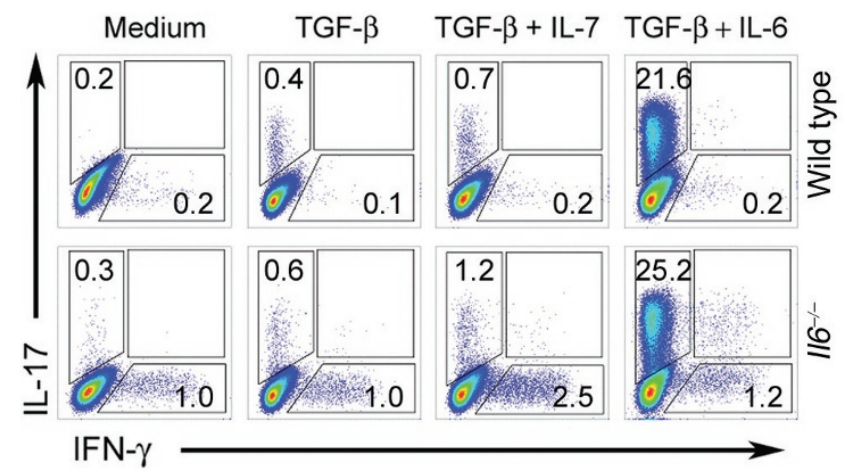

Figure 1 